\title{
Desempenho e Predição de Híbridos e Análise de Agrupamento de Características de Matrizes de Frangos de Corte
}

\author{
Valéria Maria Nascimento Abreu², Martinho de Almeida e Silva ${ }^{3}$, Cosme Damião $\mathrm{Cruz}^{4}$, \\ Élsio Antônio Pereira de Figueiredo ${ }^{5}$, Paulo Giovanni de Abreu ${ }^{6}$
}

RESUMO - Este trabalho foi conduzido com cruzamentos (dialelo parcial) entre as linhas do grupo I (LL, TL, ZL, TT, LT, ZT, ZZ, LZ e TZ) e as linhas do grupo II (PP, KP, VP, KK, PK, VK, VV, PV e KV), além de duas linhas (LLc e PPc) controle. O delineamento experimental foi em blocos casualizados consistindo de três blocos, 82 cruzamentos e nove fêmeas por cruzamento. As características de produção, no total de onze, foram: fertilidade na $32^{\mathrm{a}}, 38^{\mathrm{a}}, 42^{\mathrm{a}}$ e $49^{\mathrm{a}}$ semanas, eclodibilidade na $32^{\mathrm{a}}$ semana, peso inicial dos pintinhos, peso no $42^{\circ}$ dia, conversão no $42^{\circ}$ dia, rendimento de carcaça, peito e gordura abdominal no $42^{\circ}$ dia de idade dos frangos. As médias e predição dos híbridos mostraram ser fonte importante de informações para o melhorista. Existe similaridade entre os cruzamentos oriundos da utilização da linha 1 , como progenitor.

Palavras-chave: análise de agrupamento, desempenho, híbridos, matrizes de frango de corte, predição

\section{Performance and Prediction and Clustering Analyses of Characteristics of Parental Broiler Lines of Production Traits}

ABSTRACT - This study was conducted using data from single crosses (partial diallel) of male lines, group I (LL, TL, ZL, TT, LT, ZT, ZZ, LZ, TL), female lines, group II (PP, KP, VP, KK, PK, VK, VV, PV, KV), besides two control lines (LLc and PPc). A completely randomized block design, with three replicales, 82 single crosses treatments with nine females for experimental unit was used in the analyses. The production traits analyzed, eleven total, were: fertility at $32^{\text {nd }}, 38^{\text {th }}, 42^{\text {nd }}$ and $49^{\text {th }}$ weeks of age, hatchability at $32^{\text {nd }}$, body weight at day of age, body weight and feed:gain ratio at $42^{\text {nd }}$ days of age, and carcass yield, breast yield and fast pad yield at $42^{\text {nd }}$ day of age. The averages and prediction of the hybrid ones showed to be important source of information. Similarity exists among the crossings originating from of the use of the line 1 , as progenitor.

Key Words: clustering analyses, broiler parental lines, hybrids, performance, prediction

\section{Introdução}

O estudo do desempenho de híbridos por meio da média de características permite avaliar a possibilidade de uso per si dos híbridos avaliados, considerando para isto, fundamentalmente, a média fenotípica e o grau de complementariedade genética de seus progenitores, expressos pelos efeitos da capacidade específica de combinação. Ao se selecionarem progenitores para compor programa de cruzamentos, aproveitando as informações providas pela capacidade específica de combinação, inicialmente devem-se observar dentro dos progenitores os que apresentam maior média e, depois, os de maior capacidade geral de combinação.
De acordo com Cruz \& Regazzi (1994), a predição de híbridos, sejam duplos, triplos ou múltiplos, tem sido de grande utilidade, sendo oriunda das informações originadas pelos cruzamentos em dialelos parciais.

O híbrido triplo é o resultado do cruzamento entre o híbrido simples e uma terceira linhagem. $\mathrm{Na}$ situação mais freqüente, o híbrido simples é obtido com as linhagens de um mesmo grupo e o triplo do cruzamento desse híbrido simples com uma linhagem de outro grupo.

O híbrido duplo é o resultado do cruzamento entre dois híbridos simples. Geralmente, os híbridos simples são obtidos do cruzamento entre linhagens de determinado grupo, e o híbrido duplo, do cruzamento entre híbridos simples de grupos distintos.

\footnotetext{
${ }_{1}^{1}$ Parte do trabalho de tese de D.S. do primeiro autor, financiada pela CAPES/UFV/EMBRAPA.

${ }^{2}$ Pesquisadora EMBRAPA/CNPSA, bolsista da CAPES. Embrapa Suínos e Aves, CP. 21. Concórdia - SC. CEP. $89700-000$. E.mail: valeria@cnpsa.embrapa.br

${ }^{3}$ Professor da Universidade Federal de Minas Gerais.

${ }^{4}$ Professor da Universidade Federal de Viçosa.

5 Pesquisador EMBRAPA/CNPSA, bolsista do CNPq. E.mail: elsio@cnpsa.embrapa.br

${ }^{6}$ Pesquisador EMBRAPA/CNPSA. E.mail: pabreu@cnpsa.embrapa.br
} 
A análise de agrupamento tem por finalidade reunir, por algum critério de classificação, os progenitores (ou qualquer outro tipo de unidade amostral) em vários grupos, de tal forma que existam homogeneidade dentro do grupo e heterogeneidade entre grupos. Alternativamente, as técnicas de análise de agrupamento têm por objetivo, ainda, dividir um grupo original de observações em vários grupos, segundo algum critério de similaridade ou dissimilaridade. O processo de agrupamento envolve, basicamente, duas etapas, a primeira relaciona-se com a estimação de uma medida de similaridade (ou dissimilaridade) entre os progenitores e a segunda, com a adoção de uma técnica de agrupamento para a formação dos grupos (Cruz \& Regazzi, 1994).

Segundo Freitas (1996), a estimação das medidas de dissimilaridade consiste na conversão da matriz de observações $n \times p$ (sendo $n$ o número de indivíduos ou progenitores e p o de variáveis), em uma matriz quadrada e simétrica, $\mathrm{n} \times \mathrm{n}$, de similaridade individuais, que são medidas da distância entre pares de indivíduos, dado o valor de um conjunto de $\mathrm{p}$ variáveis. Na interseção da iésima linha e k-ésima coluna dessa matriz, coloca-se a distância entre o i-ésimo e o k-ésimo indivíduo. A alta similaridade (pequena distância) indica que os indivíduos são próximos em relação ao conjunto de variáveis, enquanto que a dissimilaridade (grande distância) indica o contrário.

Nos estudos da divergência genética destinados à identificação de progenitores para hibridação, têm sido de uso mais rotineiro a distância euclidiana média ou a distância generalizada de Mahalanobis, sendo essaúltima a preferida, entretanto, possível de ser estimada apenas quando se dispõe da matriz de covariâncias residuais estimada a partir de ensaios experimentais com repetições (Cruz \& Regazzi, 1994).

Segundo Cruz (1990), a distância euclidiana, ou o seu quadrado, é bastante utilizada quando as unidades para cálculo são escores de componentes principais ou de variáveis canônicas. Quando é estimada a partir de variáveis originais, apresenta a inconveniência de ser influenciada, além do número e da escala, pelas correlações entre aquelas variáveis. Para contornar a influência do número de variáveis sobre as estimativas da distância euclidiana, recomenda-se a utilização da distância euclidiana média; problemas inerentes à escala são contornados pela padronização prévia das variáveis em estudo. Assim, o maior inconveniente é mesmo a pressuposição da independência entre variáveis. Entretanto, Arunachalam (1981) argumenta que, nos estudos sobre divergência genética, vários caracteres com graus significativos de correlação são avaliados, desaconselhando a quantificação da divergência pela distância euclidiana e citando, como maneira alternativa para avaliar a divergência, a estatística $D^{2}$ de Mahalanobis, que leva em consideração as correlações entre características analisadas por meio da matriz de covariâncias residuais entre variáveis.

A distância generalizada de Mahalanobis considera a variabilidade de cada unidade experimental e não somente a medida de tendência amostral, sendo recomendada para dados provenientes de delineamentos e quando as características são correlacionadas. Considera, também, a correlação entre as características analisadas por meio da matriz de dispersão dessas. Quando as correlações entre as características são nulas e as características padronizadas, a estatística D2 é equivalente à distância euclidiana (Cruz, 1990).

Segundo Cruz \& Regazzi (1994), quando se dispõe de várias características, o valor de $\mathrm{D}^{2}$ pode ser, alternativamente, estimado a partir das médias dos dados originais e da matriz de covariâncias residuais, ou a partir de dados transformados. Na transformação dos dados, equivalente ao processo de rotação de eixos, são obtidas combinações lineares das características originais, cujos coeficientes de ponderação são derivados de um processo denominado condensação pivotal. A condensação pivotal é aplicada na matriz de dispersão e evita a inversão de matrizes, que, quando de ordem elevada, proporciona grandes erros numéricos. Com isso, torna os cálculos mais simples (Rao, 1952). Com o avanço dos computadores, já é possível a inversão dessas matrizes sem a necessidade da condensação pivotal. E quando se aplica a condensação pivotal, nas variáveis originais, são estimadas as médias das variáveis transformadas que têm variâncias residuais unitárias e covariâncias residuais nulas, que são variáveis não-correlacionadas, permitindo-se, assim, a estimação de $\mathrm{D}^{2}$ de maneira análoga ao quadrado da distância euclidiana.

A segunda etapa do processo de agrupamento consiste na escolha de um método de agrupamento. Dentre os métodos de agrupamento, os mais utilizados, em estudos de divergência genética, são os hierárquicos e os de otimização, sendo que o método de otimização apresentado por Tocher vem sendo extensivamente usado nesses estudos (Piassi, 1994).

Segundo Cruz \& Regazzi (1994), no método de Tocher, adota-se o critério de que a média das medidas de dissimilaridade, dentro de cada grupo, deve ser menor que as distâncias médias entre quaisquer grupos. $\mathrm{O}$ 
método requer a obtenção da matriz de dissimilaridade, sobre a qual é identificado o par de progenitores mais similar. Esses progenitores formarão o grupo inicial e, a partir daí, será avaliada a possibilidade de inclusão de novos progenitores, adotando-se o critério anteriormente citado. A entrada de um progenitor em um grupo sempre aumenta o valor médio da distância dentro do grupo. Assim, pode-se tomar a decisão de incluir o progenitor em um grupo por meio da comparação entre o acréscimo no valor médio da distância dentro do grupo e um nível máximo permitido, que pode ser estabelecido arbitrariamente, ou adotar, como tem sido geralmente feito, o valor máximo da medida de dissimilaridade encontrado das menores distâncias envolvendo cada progenitor.

Este trabalho teve como objetivo obter resultados de desempenho e predição de híbridos resultantes do cruzamento entre linhagens de matrizes de frango de corte, bem como estudar a diversidade genética, por meio da análise de agrupamento, destes cruzamentos.

\section{Material e Métodos}

Os dados foram provenientes de experimento realizado no Setor de Melhoramento Genético de Aves (SMGA) do Centro Nacional de Pesquisa de Suínos e Aves (CNPSA), da Empresa Brasileira de Pesquisa Agropecuária (EMBRAPA), em Concórdia, Santa Catarina, de janeiro de 1994 a dezembro de 1995.

Os pintos de um dia de idade deste experimento foram obtidos de cruzamentos (dialelo parcial) entre as linhas do grupo I (LL, TL, ZL, TT, LT, ZT, ZZ, LZ e TZ) e as linhas do grupo II (PP, KP, VP, KK, PK, VK, VV, PV e KV), além de duas linhas (LLc e PPc) controle.

A codificação das linhagens foi feita da seguinte maneira:

\begin{tabular}{|cc|}
\hline \multicolumn{2}{|c|}{ Linhas } \\
\cline { 2 - 3 } Gupo I & Grupo II \\
\hline $1=\mathrm{LL}$ & $1=\mathrm{PP}$ \\
$2=\mathrm{TL}$ & $2=\mathrm{KP}$ \\
$3=\mathrm{ZL}$ & $3=\mathrm{VP}$ \\
$4=\mathrm{TT}$ & $4=\mathrm{KK}$ \\
$5=\mathrm{LT}$ & $5=\mathrm{PK}$ \\
$6=\mathrm{ZT}$ & $6=\mathrm{VK}$ \\
$7=\mathrm{ZZ}$ & $7=\mathrm{VV}$ \\
$8=\mathrm{LZ}$ & $8=\mathrm{PV}$ \\
$9=\mathrm{TZ}$ & $9=\mathrm{KV}$ \\
$0=\mathrm{LLc}$ & $0=\mathrm{PPc}$ \\
\hline
\end{tabular}

- Cruzamentos

\begin{tabular}{|lllll|}
\hline $11=\mathrm{LLPP}$ & $21=\mathrm{TLPP}$ & $31=\mathrm{ZLPP}$ & $41=\mathrm{TTPP}$ & $51=\mathrm{LTPP}$ \\
$12=\mathrm{LLKP}$ & $22=\mathrm{TLKP}$ & $32=\mathrm{ZLKP}$ & $42=\mathrm{TTKP}$ & $52=\mathrm{LTKP}$ \\
$13=\mathrm{LLVP}$ & $23=\mathrm{TLVP}$ & $33=\mathrm{ZLVP}$ & $43=\mathrm{TTVP}$ & $53=\mathrm{LTVP}$ \\
$14=\mathrm{LLKK}$ & $24=\mathrm{TLKK}$ & $34=\mathrm{ZLKK}$ & $44=\mathrm{TTKK}$ & $54=\mathrm{LTKK}$ \\
$15=\mathrm{LLPK}$ & $25=\mathrm{TLPK}$ & $35=\mathrm{ZLPK}$ & $45=\mathrm{TTPK}$ & $55=\mathrm{LTPK}$ \\
$16=\mathrm{LLVK}$ & $26=\mathrm{TLVK}$ & $36=\mathrm{ZLVK}$ & $46=\mathrm{TTVK}$ & $56=\mathrm{LTVK}$ \\
$17=\mathrm{LLVV}$ & $27=\mathrm{TLVV}$ & $37=\mathrm{ZLVV}$ & $47=\mathrm{TTVV}$ & $57=\mathrm{LTVV}$ \\
$18=\mathrm{LLPV}$ & $28=\mathrm{TLPV}$ & $38=\mathrm{ZLPV}$ & $48=\mathrm{TTPV}$ & $58=\mathrm{LTPV}$ \\
$19=\mathrm{LLKV}$ & $29=\mathrm{TLKV}$ & $39=\mathrm{ZLKV}$ & $49=\mathrm{TTKV}$ & $59=\mathrm{LTKV}$ \\
\hline $61=\mathrm{ZTPP}$ & $71=\mathrm{ZZPP}$ & $81=\mathrm{LZPP}$ & $91=\mathrm{TZPP}$ & \\
$62=\mathrm{ZTKP}$ & $72=\mathrm{ZZKP}$ & $82=\mathrm{LZKP}$ & $92=\mathrm{TZKP}$ & \\
$63=\mathrm{ZTVP}$ & $73=\mathrm{ZZVP}$ & $83=\mathrm{LZVP}$ & $93=\mathrm{TZVP}$ & \\
$64=\mathrm{ZTKK}$ & $74=\mathrm{ZZKK}$ & $84=\mathrm{LZKK}$ & $94=\mathrm{TZKK}$ & \\
$65=\mathrm{ZTPK}$ & $75=\mathrm{ZZPK}$ & $85=\mathrm{LZPK}$ & $95=\mathrm{TZPK}$ & \\
$66=\mathrm{ZTVK}$ & $76=\mathrm{ZZVK}$ & $86=\mathrm{LZVK}$ & $96=\mathrm{TZVK}$ & \\
$67=\mathrm{ZTVV}$ & $77=\mathrm{ZZVV}$ & $87=\mathrm{LZVV}$ & $97=\mathrm{TZVV}$ & \\
$68=\mathrm{ZTPV}$ & $78=\mathrm{ZZPV}$ & $88=\mathrm{LZPV}$ & $98=\mathrm{TZPV}$ & \\
$69=\mathrm{ZTKV}$ & $79=\mathrm{ZZKV}$ & $89=\mathrm{LZKV}$ & $99=\mathrm{TZKV}$ & \\
\hline
\end{tabular}

$00=\operatorname{LLcPPc}($ Controle $)$

As fêmeas foram alojadas em gaiolas individuais perfazendo um total de 2.214 fêmeas. Os machos foram alojados em 60 gaiolas individuais, com piso ripado, para coleta de sêmen, destinado à fertilização posterior, via inseminação artificial, em delineamento experimental em blocos casualizados, consistindo de três blocos, 82 cruzamentos e nove fêmeas por cruzamento.

A inseminação artificial foi efetuada com 0,05 mL de um "pool" de sêmen fresco, não diluído, uma vez por semana, diretamente na vagina da galinha ( $2 \mathrm{~cm}$ de profundidade), com seringa graduada, conforme procedimento padrão utilizado no CNPSA.

Os ovos foram coletados diariamente e armazenados, a $12^{\circ} \mathrm{C}$, por sete dias antes da incubação semanal normal para a venda de pintos, com a identificação da galinha, do tratamento e da data de postura. Todos os ovos foram submetidos à ovoscopia aos 19 dias de incubação

As 11 características de produção estudadas, na análise de agrupamento e estudo do desempenho dos híbridos foram: fertilidade na $42^{\mathrm{a}}$ (FERT42); 49 (FERT49); 32 ${ }^{\mathrm{a}}$ (FERT32) e $38^{\mathrm{a}}$ (FERT38); eclodibilidade na $32^{\text {a }}$ (ECLO32); peso inicial do pintinho (PI); peso dos frangos no $42^{\circ}$ dia de idade (P42); conversão alimentar dos frangos no $42^{\circ}$ dia de idade (CA); rendimento de carcaça dos frangos no 
$42^{\circ}$ dia de idade (RCAR); rendimento de peito dos frangos no $42^{\circ}$ dia de idade (RPEIT); rendimento de gordura abdominal dos frangos no $42^{\circ}$ dia de idade (RGA).

Para a predição de híbridos foram utilizadas apenas as características: fertilidade na $38^{\mathrm{a}}$ (FERT38); peso dos frangos no $42^{\circ}$ dia de idade (P42) e rendimento de carcaça dos frangos no $42^{\circ}$ dia de idade (RCAR).

As análises foram realizadas utilizando-se o programa Genes (Cruz, 1997).

Este trabalho foi desenvolvido seguindo as etapas descritas:

a) Avaliação das médias

Este estudo foi feito levando-se em consideração as médias dos cruzamentos;

\section{b) Predição de híbridos triplos e duplos}

Um dos processos de predição baseia-se na expressão geral, de acordo com Cruz \& Regazzi (1994):

$$
\mathrm{M}=[\mathrm{X}][\mathrm{Y}]
$$

em que: $\mathrm{M}=$ média a ser predita; $[\mathrm{X}]=$ contribuição dos progenitores para o conjunto gênico que é repassado pelos gametas masculinos; $[\mathrm{Y}]=$ contribuição dos progenitores para o conjunto gênico que é repassado pelos gametas femininos.

\section{c) Análise multivariada}

A análise de agrupamento foi feita, adotando-se a distância euclidiana média como medida de dissimilaridade, sobre a qual empregou-se o método de agrupamento de otimização de Tocher.

\section{c1) Medida de dissimilaridade}

A distância euclidiana média foi utilizada para contornar a influência do número de caracteres e, para contornar o problema da escala dos dados, foi feita padronização dos dados da seguinte maneira:

$$
x_{i j}=\frac{\mathrm{X}_{\mathrm{ij}}}{\mathrm{S}\left(\mathrm{X}_{\mathrm{j}}\right)},
$$

em que: $X_{i j}$ é a observação no progenitor $\mathrm{i}(\mathrm{i}=1,2, \ldots$, $\mathrm{p})$, em referência ao caráter $\mathrm{j}(\mathrm{j}=1,2, \ldots, \mathrm{n})$; $\mathrm{S}\left(\mathrm{X}_{\mathrm{j}}\right)$ é o desvio-padrão dos dados do caráter $\mathrm{j}$.

Então, a distância euclidiana média entre dois progenitores i e i' foi dada por:

$$
d_{i i^{\prime}}=\sqrt{\frac{1}{\mathrm{n}} \sum_{\mathrm{j}}\left(\mathrm{X}_{\mathrm{ij}}-\mathrm{X}_{\mathrm{i}^{\prime} \mathrm{j}}\right)^{2}}
$$

em que: di i'é distância euclidiana média baseada em dados padronizados; $\mathrm{n}$ é o número de caracteres analisados.

\section{c2) Método de otimização de Tocher}

Um dos métodos de agrupamento mais indicados para estudar o grau de semelhança existente entre genótipos é o método de otimização de Tocher, que é utilizado na formação dos conjuntos de genótipos com maior semelhança entre si (Piassi, 1994).

Para o agrupamento segundo o método de Tocher, foi adotado o critério do maior entre os mínimos, ou seja, o acréscimo médio na distância intracluster (intragrupo), pela inclusão de um novo cruzamento a um dado cluster (grupo), deveria ser menor ou igual ao limite adotado. Estabeleceu-se que esse limite seria o valor máximo da medida de dissimilaridade encontrada no conjunto composto pelas menores distâncias de cada cruzamento a qualquer outro, na matriz de distâncias original. O acréscimo médio no valor da distância intragrupo foi calculado, dividindo-se a distância entre o cruzamento a ser inserido e o grupo que poderia recebê-lo pelo número de cruzamentos do grupo.

A matriz de dissimilaridade adotada foi a de distância euclidiana média, na qual se identificou, inicialmente, o par de cruzamentos que apresentava o menor valor de distância, para formar o primeiro grupo, quando essa distância não superava o limite estabelecido. Na seqüência, de acordo com o critério adotado, avaliou-se a possibilidade de inclusão de outros cruzamentos no grupo ou se haveria necessidade de formação de outros grupos, seguindo o mesmo critério.

Esquematicamente, mostra-se o critério para formação do agrupamento:

$$
d_{(i j) k}^{2}=d_{i k}^{2}+d_{j k}^{2} \text { ou } d_{(i j k) 1}^{2}=d_{(i j) 1}^{2}+d_{k l}^{2} ;
$$

então, se $\frac{\mathrm{d}^{2}(\text { Grupo)i }}{\mathrm{n}} \leq \alpha \Rightarrow$ inclui - se o cruzamento i no grupo; ou $\quad s e \frac{\mathrm{d}^{2}(\mathrm{Grupo}) \mathrm{i}}{\mathrm{n}}>\alpha \Rightarrow$ não inclui - se o cruzamento i no grupo em que: $\alpha=$ limite de acréscimo adotado; $\mathrm{j}, \mathrm{k}, 1=$ cruzamentos do grupo; $\mathrm{i}=$ cruzamento a ser incluído, ou não, no grupo; $\mathrm{n}$ = número de cruzamentos do grupo que poderá receber $\mathrm{i}$.

\section{Resultados e Discussão}

$\mathrm{Na}$ Tabela 1, estão apresentadas as médias das características em estudo, de acordo com os cruzamentos, incluindo-se, também, os valores do cruzamento testemunha.

A média é uma variável importante, pois, ao se

\section{R. Bras. Zootec., v.31, n.2, p.617-626, 2002}


Tabela 1 - Médias das características produtivas, de acordo com os cruzamentos Table 1 - Averages of the characteristics, in agreement with the crosses

\begin{tabular}{|c|c|c|c|c|c|c|c|c|c|c|c|}
\hline \multirow{3}{*}{$\begin{array}{l}\text { Cruzamentos } \\
\text { Crosses }\end{array}$} & \multicolumn{11}{|c|}{$\begin{array}{l}\text { Médias } \\
\text { Averages }\end{array}$} \\
\hline & FERT42 & FERT49 & FERT32 & FERT38 & ECLO32 & PI & P42 & $\mathrm{CA}$ & RCAR & RPEIT & RGA \\
\hline & FERT42 & FERT49 & FERT32 & FERT38 & HАТС 32 & $W I$ & $W 42$ & $F / G$ & $Y C A R$ & YBRE & $Y A F$ \\
\hline 11 & 88,89 & 100,00 & 90,77 & 91,67 & 86,15 & 45 & 1820 & 2,0595 & 0,67149 & 0,19467 & 0,022771 \\
\hline 12 & 100,00 & 83,33 & 90,77 & 97,92 & 78,46 & 45 & 2118 & 1,9528 & 0,70986 & 0,19703 & 0,023842 \\
\hline 13 & 100,00 & 100,00 & 90,77 & 95,83 & 87,69 & 44 & 1882 & 1,9784 & 0,69501 & 0,19636 & 0,025898 \\
\hline 14 & 92,86 & 100,00 & 89,23 & 91,67 & 86,15 & 45 & 2064 & 1,9682 & 0,72716 & 0,19925 & 0,026335 \\
\hline 15 & 94,44 & 93,75 & 95,38 & 93,75 & 84,61 & 44 & 2071 & 1,8157 & 0,72588 & 0,20422 & 0,027890 \\
\hline 16 & 70,00 & 93,33 & 92,31 & 81,25 & 89,23 & 46 & 1979 & 1,9185 & 0,69743 & 0,19601 & 0,025872 \\
\hline 17 & 92,31 & 94,12 & 92,31 & 89,58 & 83,08 & 46 & 1787 & 2,0734 & 0,70605 & 0,17472 & 0,023418 \\
\hline 18 & 88,23 & 93,75 & 90,77 & 93,75 & 86,15 & 47 & 2003 & 1,9478 & 0,69725 & 0,18372 & 0,028438 \\
\hline 19 & 89,47 & 100,00 & 93,85 & 93,75 & 86,15 & 45 & 1984 & 1,9957 & 0,72042 & 0,20260 & 0,021787 \\
\hline 21 & 80,00 & 100,00 & 93,85 & 89,58 & 81,54 & 45 & 1837 & 1,9510 & 0,71742 & 0,20069 & 0,01792 \\
\hline 22 & 100,00 & 92,31 & 92,31 & 93,75 & 84,61 & 45 & 2006 & 2,0262 & 0,70569 & 0,20751 & 0,021878 \\
\hline 23 & 83,33 & 90,91 & 96,92 & 91,67 & 90,77 & 46 & 1801 & 2,0196 & 0,71734 & 0,21171 & 0,017364 \\
\hline 24 & 75,00 & 92,86 & 93,85 & 89,58 & 86,15 & 43 & 2119 & 1,9867 & 0,72945 & 0,20434 & 0,023813 \\
\hline 25 & 69,23 & 87,50 & 92,31 & 89,58 & 83,08 & 47 & 1969 & 1,9774 & 0,71847 & 0,20865 & 0,024001 \\
\hline 26 & 89,47 & 91,67 & 87,69 & 81,25 & 75,38 & 47 & 1867 & 1,9773 & 0,69910 & 0,18608 & 0,027556 \\
\hline 27 & 100,00 & 88,89 & 86,15 & 95,83 & 75,38 & 47 & 2034 & 1,8666 & 0,72932 & 0,21115 & 0,020156 \\
\hline 28 & 86,36 & 92,31 & 90,77 & 87,50 & 87,69 & 45 & 1912 & 2,0590 & 0,70547 & 0,18672 & 0,021176 \\
\hline 29 & 94,74 & 100,00 & 87,69 & 91,67 & 83,08 & 46 & 1925 & 2,0276 & 0,72947 & 0,20359 & 0,035342 \\
\hline 31 & 78,57 & 92,31 & 92,31 & 97,92 & 86,15 & 47 & 1936 & 1,8613 & 0,72145 & 0,18947 & 0,032582 \\
\hline 32 & 88,23 & 100,00 & 90,77 & 81,25 & 84,61 & 46 & 2080 & 1,9308 & 0,71476 & 0,19475 & 0,018246 \\
\hline 33 & 56,25 & 91,67 & 89,23 & 93,75 & 81,54 & 45 & 1963 & 1,9327 & 0,69297 & 0,19444 & 0,018258 \\
\hline 34 & 88,23 & 91,67 & 95,39 & 89,58 & 84,61 & 45 & 2035 & 1,9200 & 0,71328 & 0,20818 & 0,018395 \\
\hline 35 & 89,47 & 100,00 & 86,15 & 91,67 & 76,92 & 47 & 1884 & 2,0186 & 0,71152 & 0,19781 & 0,019100 \\
\hline 36 & 87,50 & 83,33 & 92,31 & 93,75 & 86,15 & 48 & 1952 & 1,8832 & 0,72323 & 0,17776 & 0,041254 \\
\hline 37 & 83,33 & 95,45 & 92,31 & 91,67 & 86,15 & 46 & 1929 & 2,0432 & 0,72489 & 0,19906 & 0,022857 \\
\hline 38 & 92,31 & 100,00 & 92,31 & 97,92 & 86,15 & 46 & 1878 & 1,8913 & 0,69648 & 0,20066 & 0,013912 \\
\hline 39 & 83,33 & 76,92 & 87,69 & 95,83 & 80,00 & 47 & 2031 & 1,8863 & 0,72087 & 0,20969 & 0,024927 \\
\hline 41 & 86,67 & 100,00 & 93,85 & 91,67 & 86,15 & 42 & 2001 & 1,9204 & 0,71509 & 0,20291 & 0,018609 \\
\hline 42 & 100,00 & 92,31 & 89,23 & 93,62 & 81,54 & 45 & 2147 & 1,9294 & 0,75395 & 0,21319 & 0,025675 \\
\hline 43 & 100,00 & 86,67 & 86,15 & 95,35 & 76,92 & 44 & 2026 & 1,9346 & 0,72926 & 0,21758 & 0,024902 \\
\hline 44 & 81,82 & 100,00 & 92,31 & 85,71 & 87,69 & 45 & 2252 & 1,7813 & 0,73140 & 0,21379 & 0,023571 \\
\hline 45 & 85,71 & 100,00 & 93,85 & 91,30 & 86,15 & 46 & 1975 & 1,9022 & 0,70744 & 0,21549 & 0,019794 \\
\hline 46 & 100,00 & 91,67 & 86,15 & 91,67 & 80,00 & 47 & 1970 & 1,9423 & 0,60397 & 0,17882 & 0,014605 \\
\hline 47 & 83,33 & 83,33 & 96,92 & 83,33 & 87,69 & 46 & 2029 & 1,9668 & 0,71438 & 0,19237 & 0,029083 \\
\hline 48 & 77,78 & 100,00 & 88,89 & 91,67 & 82,54 & 46 & 2071 & 1,8799 & 0,71354 & 0,21138 & 0,016782 \\
\hline 49 & 92,85 & 100,00 & 95,38 & 80,85 & 84,61 & 46 & 2065 & 1,8631 & 0,73528 & 0,20517 & 0,026050 \\
\hline 51 & 92,86 & 90,00 & 90,77 & 89,58 & 80,00 & 44 & 1970 & 1,8753 & 0,72372 & 0,19659 & 0,024588 \\
\hline 52 & 86,36 & 71,49 & 90,77 & 89,58 & 75,38 & 46 & 2081 & 1,8792 & 0,73174 & 0,21704 & 0,018988 \\
\hline 53 & 69,23 & 100,00 & 92,19 & 83,33 & 79,69 & 45 & 1937 & 1,9380 & 0,71916 & 0,19626 & 0,019434 \\
\hline 54 & 73,33 & 88,23 & 92,31 & 83,33 & 81,54 & 45 & 2377 & 1,8750 & 0,71707 & 0,20423 & 0,020874 \\
\hline 55 & 100,00 & 76,92 & 96,92 & 91,67 & 83,08 & 46 & 2012 & 1,9573 & 0,72384 & 0,20407 & 0,028660 \\
\hline 56 & 92,31 & 87,50 & 96,92 & 89,58 & 89,23 & 46 & 1957 & 1,9902 & 0,70349 & 0,19634 & 0,017396 \\
\hline 57 & 70,83 & 93,75 & 90,62 & 95,83 & 82,81 & 44 & 1898 & 1,9488 & 0,71331 & 0,21330 & 0,021666 \\
\hline
\end{tabular}




\section{Continuação da Tabela $1 . .$.}

Tabela 1 - Médias das características produtivas, de acordo com os cruzamentos

Table 1 - Averages of the characteristics, in agreement with the crosses

\begin{tabular}{|c|c|c|c|c|c|c|c|c|c|c|c|}
\hline \multirow{3}{*}{$\begin{array}{l}\text { Cruzamentos } \\
\text { Crosses }\end{array}$} & \multicolumn{11}{|c|}{$\begin{array}{l}\text { Médias } \\
\text { Averages }\end{array}$} \\
\hline & FERT42 & FERT49 & FERT32 & FERT38 & ECLO32 & PI & P42 & $\mathrm{CA}$ & RCAR & RPEIT & RGA \\
\hline & FERT42 & FERT49 & FERT32 & FERT38 & НАТС 32 & $W I$ & W42 & $F / G$ & $Y C A R$ & $Y B R E$ & $Y A F$ \\
\hline 58 & 77,78 & 94,44 & 93,85 & 83,33 & 80,00 & 46 & 1870 & 1,9455 & 0,72298 & 0,20720 & 0,029903 \\
\hline 59 & 100,00 & 100,00 & 95,38 & 93,75 & 89,23 & 47 & 2049 & 2,0501 & 0,73217 & 0,21041 & 0,028198 \\
\hline 61 & 85,00 & 100,00 & 90,77 & 89,58 & 83,08 & 44 & 1993 & 1,8915 & 0,72539 & 0,21130 & 0,019243 \\
\hline 62 & 93,33 & 94,74 & 98,46 & 87,50 & 89,23 & 45 & 2033 & 1,8384 & 0,70235 & 0,20980 & 0,018051 \\
\hline 63 & 76,19 & 87,50 & 93,85 & 92,45 & 80,00 & 44 & 2014 & 1,9216 & 0,72559 & 0,18983 & 0,021235 \\
\hline 64 & 63,64 & 90,00 & 90,77 & 81,25 & 80,00 & 44 & 2158 & 1,8062 & 0,71882 & 0,21903 & 0,017968 \\
\hline 65 & 75,00 & 76,92 & 92,31 & 89,58 & 84,61 & 46 & 1924 & 1,8909 & 0,70068 & 0,21541 & 0,013728 \\
\hline 66 & 71,43 & 100,00 & 95,38 & 93,75 & 84,61 & 46 & 1973 & 1,8951 & 0,70395 & 0,20178 & 0,016613 \\
\hline 67 & 66,67 & 83,33 & 80,00 & 81,25 & 75,38 & 46 & 1788 & 1,9371 & 0,78171 & 0,21281 & 0,045094 \\
\hline 68 & 68,75 & 83,33 & 69,23 & 85,42 & 61,54 & 45 & 1952 & 1,9478 & 0,71170 & 0,19726 & 0,025307 \\
\hline 69 & 86,67 & 92,31 & 93,85 & 82,69 & 84,61 & 45 & 2044 & 1,9862 & 0,70973 & 0,20885 & 0,019925 \\
\hline 71 & 89,47 & 100,00 & 95,38 & 91,67 & 87,69 & 43 & 1918 & 1,9039 & 0,70911 & 0,20704 & 0,021534 \\
\hline 72 & 93,75 & 80,00 & 84,61 & 95,83 & 70,77 & 46 & 2049 & 1,9528 & 0,73768 & 0,21372 & 0,024740 \\
\hline 73 & 88,89 & 88,23 & 95,38 & 93,88 & 84,61 & 45 & 1971 & 1,9782 & 0,61030 & 0,17969 & 0,016272 \\
\hline 74 & 80,00 & 83,33 & 92,31 & 77,08 & 84,61 & 45 & 2076 & 1,8568 & 0,73318 & 0,21145 & 0,020966 \\
\hline 75 & 89,47 & 76,47 & 95,38 & 95,83 & 83,08 & 46 & 1934 & 1,9505 & 0,72434 & 0,18998 & 0,025438 \\
\hline 76 & 73,68 & 83,33 & 87,69 & 83,33 & 80,00 & 47 & 1910 & 1,9133 & 0,70774 & 0,20516 & 0,020934 \\
\hline 77 & 58,33 & 85,71 & 96,92 & 89,79 & 92,31 & 46 & 2014 & 1,7288 & 0,72699 & 0,20544 & 0,027943 \\
\hline 78 & 86,96 & 100,00 & 90,77 & 97,92 & 81,54 & 48 & 2082 & 1,8536 & 0,74520 & 0,19566 & 0,032809 \\
\hline 79 & 84,00 & 100,00 & 93,85 & 89,58 & 90,77 & 47 & 1870 & 1,9751 & 0,70799 & 0,22479 & 0,013991 \\
\hline 81 & 85,71 & 80,00 & 90,77 & 58,33 & 83,08 & 45 & 1997 & 1,9464 & 0,73632 & 0,19313 & 0,023563 \\
\hline 82 & 89,47 & 81,82 & 93,85 & 81,13 & 87,69 & 45 & 2092 & 1,9170 & 0,71664 & 0,20055 & 0,019705 \\
\hline 83 & 76,47 & 91,67 & 84,61 & 72,92 & 75,38 & 47 & 1942 & 2,0315 & 0,72888 & 0,19517 & 0,021543 \\
\hline 84 & 83,33 & 66,67 & 89,23 & 64,58 & 86,15 & 44 & 2013 & 1,8688 & 0,72931 & 0,20347 & 0,020790 \\
\hline 85 & 88,89 & 83,33 & 87,69 & 77,08 & 78,46 & 46 & 2061 & 1,9082 & 0,70718 & 0,20159 & 0,022306 \\
\hline 86 & 78,26 & 87,50 & 90,77 & 53,19 & 80,00 & 47 & 1964 & 1,9522 & 0,70263 & 0,20125 & 0,015434 \\
\hline 87 & 94,12 & 80,00 & 96,92 & 62,50 & 87,69 & 45 & 1988 & 1,8280 & 0,72000 & 0,20132 & 0,014801 \\
\hline 88 & 78,57 & 92,31 & 89,23 & 79,17 & 84,61 & 47 & 1960 & 2,0093 & 0,71999 & 0,19248 & 0,027803 \\
\hline 89 & 73,33 & 81,25 & 96,92 & 72,92 & 86,15 & 46 & 2066 & 1,9328 & 0,74251 & 0,21415 & 0,021019 \\
\hline 91 & 73,33 & 100,00 & 95,38 & 87,50 & 86,15 & 46 & 1925 & 1,9266 & 0,71264 & 0,20036 & 0,025776 \\
\hline 92 & 84,61 & 92,86 & 95,39 & 87,50 & 87,69 & 46 & 2114 & 1,9232 & 0,73990 & 0,22057 & 0,022288 \\
\hline 93 & 92,86 & 92,31 & 92,31 & 85,71 & 84,61 & 45 & 1860 & 1,9509 & 0,71972 & 0,18717 & 0,027806 \\
\hline 94 & 90,00 & 83,33 & 90,77 & 85,42 & 80,00 & 44 & 2009 & 1,9943 & 0,74800 & 0,20305 & 0,021715 \\
\hline 95 & 92,86 & 92,31 & 89,23 & 95,83 & 80,00 & 43 & 2048 & 1,8811 & 0,72242 & 0,21532 & 0,018493 \\
\hline 96 & 84,21 & 78,95 & 78,46 & 79,17 & 70,77 & 46 & 1964 & 1,9254 & 0,71505 & 0,21365 & 0,015511 \\
\hline 97 & 88,89 & 100,00 & 95,38 & 85,42 & 92,31 & 47 & 1953 & 1,9717 & 0,72107 & 0,20585 & 0,025622 \\
\hline 98 & 100,00 & 100,00 & 96,92 & 89,58 & 90,77 & 45 & 1812 & 1,8702 & 0,70602 & 0,18518 & 0,020139 \\
\hline 99 & 85,71 & 91,67 & 90,77 & 85,42 & 86,15 & 47 & 1811 & 2,0859 & 0,72602 & 0,20500 & 0,018179 \\
\hline 00 & 95,00 & 100,00 & 93,85 & 95,83 & 86,15 & 43 & 1543 & 2,0677 & 0,68891 & 0,17512 & 0,026542 \\
\hline
\end{tabular}


selecionarem progenitores para compor programa de cruzamentos, aproveitando as informações providas pela capacidade especifica de combinação, inicialmente devem-se observar dentro dos progenitores os que apresentam maior média e depois os de maior capacidade geral de combinação.

Segundo Costa (1994), são padrões de produtividade de uma boa matriz:

- peso médio, às 66 semanas, da fêmea: $3.900 \mathrm{~g}$

- ovos produzidos/ave alojada: 184,5

- eclosão média: $85,55 \%$

- fertilidade: $90 \%$

De acordo com Torres (1996), a carcaça ideal nas aves deve ser bem musculosa, livre de defeitos e com alta produção de carne. Carcaças com maior produção de carne estão associadas com menores custos de processamento e de comercialização. Frangos com menos deposição de gordura têm mostrado pequena superioridade na produção de carcaça.

Ainda segundo o mesmo autor, boa produção de ovos com alta fertilidade e eclodibilidade é importante em todos os níveis de produção de frangos de corte. Nas linhas puras, estas características permitem intensidade de seleção ótima; em avós e matrizes, permitem menor custo de ovos e de pintos.

Pelos resultados apresentados, nota-se que as características de fertilidade e eclodibilidade estão dentro do esperado, excetuando-se um ou outro cruzamento que apresentou baix as fertilidade e eclodibilidade, assim como alguns valores de $100 \%$ para fertilidade, que não condizem com a realidade, mas que podem ter surgido por problemas na amostragem. Para as características do frango de corte, em geral, os resultados dos cruzamentos são melhores quando comparados com o cruzamento controle, mostrando a superioridade dos híbridos.

O híbrido de maior valor da característica P42 é o que envolve as linhas 5, do grupo I, e 4' do grupo II. Estas linhas também haviam sido selecionadas pela maior capacidade geral de combinação em trabalho anterior. Tanto para a característica P42, como para as características RCAR e FERT38, os
20 melhores híbridos envolvem cruzamentos que contêm as linhas selecionadas, em trabalho anterior pela capacidade geral de combinação, no grupo I e II.

Dos 648 híbridos triplos possíveis, ou seja pq $(p+q-2) / 2$ híbridos apresentam-se, neste trabalho, os 20 melhores (Tabelas 2 e 3), para as características FERT38, P42 e RCAR.

O híbrido de maior valor da característica P42 é o que envolve as linhas 5, do grupo I, e 4' do grupo II. Estas linhas também haviam sido selecionadas pela maior capacidade geral de combinação em trabalho anterior. Tanto para a característica P42, como para as características RCAR e FERT38, os 20 melhores híbridos envolvem cruzamentos que contêm as linhas selecionadas, em trabalho anterior pela capacidade geral de combinação, nos grupos I e II.

Dos 1.296 híbridos duplos possíveis neste estudo, ou seja, dos pq $(p-1)(q-1) / 4$ híbridos, são apresentados somente os 40 melhores (Tabela 4).

Também para estes híbridos, os 40 melhores híbridos duplos são compostos por cruzamentos que envolvem as linhagens anteriormente selecionadas pela capacidade geral de combinação. Estes resultados mostram que a predição de híbridos oriunda de informações dos dialelos parciais é mais uma fonte importante de informação para o melhorista.

$\mathrm{Na}$ análise de agrupamento, estes agrupamentos foram feitos usando-se como medida de dissimilaridade a distância euclidiana média. O maior entre os mínimos estabelecido pelo limite de distância - Método de Tocher - foi de 0,912 $(\alpha=0,912)$. Dessa forma, foram formados vinte e nove grupos de cruzamentos (Tabela 5), sendo que os cruzamentos foram distribuídos uniformemente dentro dos grupos, não existindo grupos com grandes concentrações de cruzamentos. Os grupos 2 e 1 foram os que apresentaram maior número de cruzamentos ( 7 e 6 , respectivamente). O grupo 1 mostra a similaridade entre os cruzamentos obtidos pelo uso da linha 1 (linha macho) como progenitor, o que não é verificado para mais nenhum grupo. 
Tabela 2 - Predição dos 20 melhores híbridos triplos para as características FERT38, P42 e RCAR (grupo I × grupo II) Table 2 - Prediction of the 20 better hybrid triples for the characteristics FERT38, W42 and WCAR (group I X group II)

\begin{tabular}{|c|c|c|c|c|c|}
\hline \multicolumn{6}{|c|}{$\begin{array}{l}\text { Grupo I } \times \text { Grupo II } \\
\text { Group I } \times \text { Group II }\end{array}$} \\
\hline \multirow{2}{*}{\multicolumn{2}{|c|}{$\begin{array}{l}\text { FERT38 } \\
\text { FERT } 38\end{array}$}} & \multirow{2}{*}{\multicolumn{2}{|c|}{$\begin{array}{l}\mathrm{P} 42 \\
W 42\end{array}$}} & \multirow{2}{*}{\multicolumn{2}{|c|}{$\begin{array}{c}\text { RCAR } \\
Y C A R\end{array}$}} \\
\hline & & & & & \\
\hline Cruzamento & Valor & Cruzamento & Valor & Cruzamento & Valor \\
\hline Crosses & Value & Crosses & Value & Crosses & Value \\
\hline $3 \times 7-8^{\prime}$ & 97,9169 & $4 \times 5-4^{\prime}$ & 2,3145 & $2 \times 6-7^{\prime}$ & 0,7555 \\
\hline $1 \times 7-2^{\prime}$ & 96,8750 & $5 \times 6-4^{\prime}$ & 2,2675 & $6 \times 7-7^{\prime}$ & 0,7543 \\
\hline $1 \times 7-8^{\prime}$ & 95,8335 & $2 \times 5-4^{\prime}$ & 2,2480 & $3 \times 6-7^{\prime}$ & 0,7533 \\
\hline $1 \times 3-8^{\prime}$ & 95,8335 & $5 \times 7-4^{\prime}$ & 2,2265 & $6 \times 9-7^{\prime}$ & 0,7514 \\
\hline $1 \times 2-2^{\prime}$ & 95,8334 & $1 \times 5-4^{\prime}$ & 2,2205 & $6 \times 8-7^{\prime}$ & 0,7508 \\
\hline $7 \times 9-5^{\prime}$ & 95,8330 & $5 \times 8-4^{\prime}$ & 2,1950 & $7 \times 6-7^{\prime}$ & 0,7480 \\
\hline $2 \times 5-7^{\prime}$ & 95,8330 & $2 \times 4-4^{\prime}$ & 2,1855 & $5 \times 6-1^{\prime}$ & 0,7475 \\
\hline $1 \times 4-2^{\prime}$ & 95,7669 & $4 \times 7-4^{\prime}$ & 2,1640 & $4 \times 9-2^{\prime}$ & 0,7469 \\
\hline $1 \times 4-3^{\prime}$ & 95,5910 & $1 \times 4-4^{\prime}$ & 2,1580 & $4 \times 7-2^{\prime}$ & 0,7458 \\
\hline $1 \times 7-3^{\prime}$ & 94,8554 & $4 \times 4-4^{\prime}$ & 2,1435 & $1 \times 6-7^{\prime}$ & 0,7439 \\
\hline $3 \times 4-1^{\prime}$ & 94,7919 & $2 \times 6-4^{\prime}$ & 2,1385 & $4 \times 5-2^{\prime}$ & 0,7428 \\
\hline $3 \times 4-8^{\prime}$ & 94,7919 & $4 \times 8-4^{\prime}$ & 2,1325 & $7 \times 9-4^{\prime}$ & 0,7406 \\
\hline $3 \times 7-1^{\prime}$ & 94,7919 & $1 \times 4-2^{\prime}$ & 2,1325 & $4 \times 8-9^{\prime}$ & 0,7389 \\
\hline $4 \times 7-8^{\prime}$ & 94,7919 & $4 \times 9-2^{\prime}$ & 2,1305 & $7 \times 9-2^{\prime}$ & 0,7388 \\
\hline $1 \times 3-1^{\prime}$ & 94,7919 & $4 \times 9-4^{\prime}$ & 2,1305 & $2 \times 9-4^{\prime}$ & 0,7387 \\
\hline $1 \times 3-3^{\prime}$ & 94,7915 & $4 \times 8-2^{\prime}$ & 2,1195 & $8 \times 9-4^{\prime}$ & 0,7387 \\
\hline $1 \times 7-5^{\prime}$ & 94,7915 & $6 \times 7-4^{\prime}$ & 2,1170 & $1 \times 9-4^{\prime}$ & 0,7376 \\
\hline $1 \times 9-5^{\prime}$ & 94,7915 & $1 \times 9-2^{\prime}$ & 2,1160 & $5 \times 8-9^{\prime}$ & 0,7373 \\
\hline $2 \times 7-2^{\prime}$ & 94,7915 & $4 \times 5-2^{\prime}$ & 2,1140 & $2 \times 8-9^{\prime}$ & 0,7360 \\
\hline $3 \times 5-9^{\prime}$ & 94,7915 & $3 \times 4-2^{\prime}$ & 2,1135 & $5 \times 9-2^{\prime}$ & 0,7358 \\
\hline
\end{tabular}

Tabela 3 - Predição dos 20 melhores híbridos triplos para as características FERT38, P42 e RCAR (grupo I × grupo II) Table 3 - Prediction of the 20 better hybrid triples for the characteristics FERT38, W42 and WCAR (group I X group II)

\begin{tabular}{|c|c|c|c|c|c|}
\hline \multicolumn{6}{|c|}{$\begin{array}{l}\text { Grupo I } \times \text { Grupo II } \\
\text { Group I } \times \text { Group II }\end{array}$} \\
\hline \multicolumn{2}{|c|}{$\begin{array}{l}\text { FERT38 } \\
\text { FERT } 38\end{array}$} & \multicolumn{2}{|c|}{$\begin{array}{l}\mathrm{P} 42 \\
W 42\end{array}$} & \multicolumn{2}{|c|}{$\begin{array}{c}\mathrm{RCAR} \\
Y C A R\end{array}$} \\
\hline $\begin{array}{c}\text { Cruzamento } \\
\text { Crosses }\end{array}$ & $\begin{array}{l}\text { Valor } \\
\text { Value }\end{array}$ & $\begin{array}{c}\text { Cruzamento } \\
\text { Crosses } \\
\end{array}$ & $\begin{array}{l}\text { Valor } \\
\text { Value }\end{array}$ & $\begin{array}{c}\text { Cruzamento } \\
\text { Crosses } \\
\end{array}$ & $\begin{array}{l}\text { Valor } \\
\text { Value }\end{array}$ \\
\hline $1^{\prime} \times 8^{\prime}-3$ & 97,6169 & $2^{\prime} \times 4^{\prime}-5$ & 2,2290 & $3^{\prime} \times 7^{\prime}-6$ & 0,7536 \\
\hline 1 'x9'- 3 & 96,8750 & $4^{\prime} \times 9^{\prime}-5$ & 2,2130 & $1^{\prime} \times 7^{\prime}-6$ & 0,7535 \\
\hline $2^{\prime} \times 3^{\prime}-1$ & 96,8750 & $2^{\prime} \times 4^{\prime}-4$ & 2,1995 & $4^{\prime} \times 7^{\prime}-6$ & 0,7503 \\
\hline $2^{\prime} \times 8^{\prime}-7$ & 96,8750 & $4^{\prime} \times 5^{\prime}-5$ & 2,1945 & $7^{\prime} \times 8^{\prime}-6$ & 0,7467 \\
\hline $5^{\prime} \times 8^{\prime}-7$ & 96,8750 & $1 ' x 4^{\prime}-5$ & 2,1735 & $2^{\prime} \times 9^{\prime}-4$ & 0,7446 \\
\hline $8^{\prime} \times 9^{\prime}-3$ & 96,8750 & $4^{\prime} \times 6^{\prime}-5$ & 2,1670 & $2^{\prime} \times 4^{\prime}-9$ & 0,7439 \\
\hline $3^{\prime} \times 8^{\prime}-7$ & 95,8974 & $4^{\prime} \times 8^{\prime}-4$ & 2,1615 & $6^{\prime} \times 7^{\prime}-6$ & 0,7428 \\
\hline $2^{\prime} \times 5^{\prime}-1$ & 95,8334 & $4^{\prime} \times 9^{\prime}-4$ & 2,1585 & $2^{\prime} \times 4^{\prime}-4$ & 0,7427 \\
\hline $2^{\prime} \times 9^{\prime}-1$ & 95,8334 & $3^{\prime} \times 4^{\prime}-5$ & 2,1570 & $2^{\prime} \times 7^{\prime}-6$ & 0,7420 \\
\hline $3^{\prime} \times 8^{\prime}-3$ & 95,8334 & $4^{\prime} \times 7^{\prime}-4$ & 2,1405 & $2^{\prime} \times 3^{\prime}-4$ & 0,7416 \\
\hline $6^{\prime} \times 8^{\prime}-3$ & 95,8334 & $3^{\prime} \times 4^{\prime}-4$ & 2,1390 & $2^{\prime} \times 7^{\prime}-6$ & 0,7420 \\
\hline $1 ' x 3$ ' - 3 & 95,8334 & $4^{\prime} \times 7^{\prime}-5$ & 2,1375 & $5^{\prime} \times 7^{\prime}-6$ & 0,7412 \\
\hline $1^{\prime} \times 6^{\prime}-3$ & 95,8334 & $1^{\prime} \times 4^{\prime}-4$ & 2,1265 & 1 'x9'- 8 & 0,7394 \\
\hline $2^{\prime} \times 8^{\prime}-1$ & 95,8334 & $4^{\prime} \times 8^{\prime}-5$ & 2,1235 & $4^{\prime} \times 8^{\prime}-7$ & 0,7392 \\
\hline $2^{\prime} \times 5^{\prime}-7$ & 95,8330 & $4^{\prime} \times 5^{\prime}-4$ & 2,1135 & $4^{\prime} \times 9^{\prime}-9$ & 0,7370 \\
\hline $3^{\prime} \times 5^{\prime}-7$ & 94,8554 & $4^{\prime} \times 6^{\prime}-4$ & 2,1110 & $7^{\prime} \times 8^{\prime}-7$ & 0,7361 \\
\hline $2^{\prime} \times 3^{\prime}-7$ & 94,8554 & $2^{\prime} \times 8^{\prime}-4$ & 2,1090 & $4^{\prime} \times 9^{\prime}-8$ & 0,7359 \\
\hline $1 ' \times 2$ '- 1 & 94,7919 & $2^{\prime} \times 9^{\prime}-4$ & 2,1060 & $3^{\prime} \times 9^{\prime}-8$ & 0,7357 \\
\hline $1 ' \times 5$ '- 3 & 94,7919 & $4^{\prime} \times 9^{\prime}-6$ & 2,1010 & $2^{\prime} \times 4^{\prime}-7$ & 0,7354 \\
\hline $1 ' x 8^{\prime}-3$ & 94,7919 & $2^{\prime} \times 4^{\prime}-6$ & 2,0955 & $4^{\prime} \times 5^{\prime}-9$ & 0,7352 \\
\hline
\end{tabular}


Tabela 4 - Predição dos 40 melhores híbridos duplos para as características FERT38, P42 e RCAR (grupo I × grupo II) Table 4 - Prediction of the 40 better hybrid double for the characteristics FERT38, W42 and WCAR (group I $\times$ group II)

\begin{tabular}{|c|c|c|c|c|c|}
\hline \multicolumn{6}{|c|}{$\begin{array}{l}\text { Grupo I } \times \text { Grupo II } \\
\text { Group } I \times \text { Group II }\end{array}$} \\
\hline \multirow{2}{*}{\multicolumn{2}{|c|}{$\begin{array}{l}\text { FERT38 } \\
\text { FERT } 38\end{array}$}} & \multirow{2}{*}{\multicolumn{2}{|c|}{$\begin{array}{l}\mathrm{P} 42 \\
W 42 \\
\end{array}$}} & \multirow{2}{*}{\multicolumn{2}{|c|}{$\begin{array}{c}\text { RCAR } \\
Y C A R\end{array}$}} \\
\hline & & & & & \\
\hline Cruzamento & Valor & Cruzamento & Valor & Cruzamento & Valor \\
\hline Crosses & Value & Crosses & Value & Crosses & Value \\
\hline $3 \times 7-1^{\prime} \times 8^{\prime}$ & 96,3544 & $4 \times 5-2^{\prime} \times 4^{\prime}$ & 2,2142 & $4 \times 9-2^{\prime} \times 4^{\prime}$ & 0,7433 \\
\hline $1 \times 7-2^{\prime} \times 8^{\prime}$ & 96,3542 & $4 \times 5-4^{\prime} \times 9^{\prime}$ & 2,1858 & $4 \times 5-4^{\prime} \times 7^{\prime}$ & 0,7424 \\
\hline $3 \times 7-3^{\prime} \times 8^{\prime}$ & 95,8655 & $5 \times 6-2^{\prime} \times 4^{\prime}$ & 2,1622 & $6 \times 7-7^{\prime} \times 8^{\prime}$ & 0,7414 \\
\hline $1 \times 7-2^{\prime} \times 3^{\prime}$ & 95,8652 & $1 \times 5-2^{\prime} \times 4^{\prime}$ & 2,1600 & $6 \times 8-1^{\prime} \times 7^{\prime}$ & 0,7409 \\
\hline $3 \times 7-5^{\prime} \times 8^{\prime}$ & 95,8334 & $5 \times 6-4^{\prime} \times 9^{\prime}$ & 2,1570 & $6 \times 7-4^{\prime} \times 7^{\prime}$ & 0,7402 \\
\hline $1 \times 7-2^{\prime} \times 5^{\prime}$ & 95,8332 & $4 \times 5-4^{\prime} \times 5^{\prime}$ & 2,1540 & $2 \times 6-4^{\prime} \times 7^{\prime}$ & 0,7398 \\
\hline $1 \times 4-2^{\prime} \times 3^{\prime}$ & 95,6790 & $4 \times 5-1 ' x 4^{\prime}$ & 2,1500 & $7 \times 9-2 ’ x 4^{\prime}$ & 0,7397 \\
\hline $1 \times 7-3^{\prime} \times 8^{\prime}$ & 95,3444 & $4 \times 5-3^{\prime} \times 4^{\prime}$ & 2,1480 & $4 \times 7-2^{\prime} \times 4^{\prime}$ & 0,7391 \\
\hline $1 \times 3-1 ' x 8^{\prime}$ & 95,3127 & $4 \times 6-2^{\prime} \times 4^{\prime}$ & 2,1475 & $6 \times 8-3^{\prime} \times 7^{\prime}$ & 0,7390 \\
\hline $1 \times 3-3^{\prime} \times 8^{\prime}$ & 95,3125 & $5 \times 7-2^{\prime} \times 4^{\prime}$ & 2,1458 & $4 \times 9-2^{\prime} \times 9^{\prime}$ & 0,7388 \\
\hline $1 \times 7-5^{\prime} \times 8^{\prime}$ & 95,3125 & $2 \times 5-2^{\prime} \times 4^{\prime}$ & 2,1457 & $2 \times 6-1 ' x 7^{\prime}$ & 0,7385 \\
\hline $1 \times 3-8^{\prime} \times 9^{\prime}$ & 95,3125 & $5 \times 9-2^{\prime} \times 4^{\prime}$ & 2,1452 & $6 \times 8-4^{\prime} \times 7^{\prime}$ & 0,7385 \\
\hline $3 \times 7-8^{\prime} \times 9^{\prime}$ & 95,3125 & $1 \times 4-2^{\prime} \times 4^{\prime}$ & 2,1452 & $2 \times 6-3^{\prime} \times 7^{\prime}$ & 0,7385 \\
\hline $1 \times 7-3^{\prime} \times 5^{\prime}$ & 94,8235 & $3 \times 5-2^{\prime} \times 4^{\prime}$ & 2,1432 & $3 \times 6-1 ' x 7^{\prime}$ & 0,7384 \\
\hline $1 \times 3-1 ' x 7^{\prime}$ & 94,7917 & $4 \times 5-4^{\prime} \times 8^{\prime}$ & 2,1425 & $4 \times 5-2^{\prime} \times 9^{\prime}$ & 0,7383 \\
\hline 1x3-1'x3' & 94,7917 & $5 \times 8-2^{\prime} \times 4^{\prime}$ & 2,1407 & $4 \times 6-2^{\prime} \times 7^{\prime}$ & 0,7381 \\
\hline $1 \times 2-2^{\prime} \times 3^{\prime}$ & 94,7917 & $4 \times 5-4^{\prime} \times 6{ }^{\prime}$ & 2,1390 & $4 \times 6-3 ’ x 7$ & 0,7377 \\
\hline $7 \times 9-5^{\prime} \times 8^{\prime}$ & 94,7915 & $4 \times 5-4^{\prime} \times 7^{\prime}$ & 2,1390 & $2 \times 6-7^{\prime} \times 9^{\prime}$ & 0,7376 \\
\hline $1 \times 3-3^{\prime} \times 9^{\prime}$ & 94,7915 & $1 \times 5-4^{\prime} \times 5^{\prime}$ & 2,1310 & $4 \times 7-2^{\prime} \times 8^{\prime}$ & 0,7376 \\
\hline $4 \times 7-2^{\prime} \times 8^{\prime}$ & 94,7584 & $2 \times 4-2^{\prime} \times 4^{\prime}$ & 2,1310 & $6 \times 8-4^{\prime} \times 7^{\prime}$ & 0,7375 \\
\hline $4 \times 7-3^{\prime} \times 8^{\prime}$ & 94,7027 & $4 \times 7-2^{\prime} \times 4^{\prime}$ & 2,1310 & $6 \times 7-2^{\prime} \times 7^{\prime}$ & 0,7372 \\
\hline $3 \times 4-1 ' x 3 '$ & 94,6707 & $4 \times 9-2^{\prime} \times 4^{\prime}$ & 2,1305 & $4 \times 8-2^{\prime} \times 9^{\prime}$ & 0,7371 \\
\hline $3 \times 4-3^{\prime} \times 8^{\prime}$ & 94,6707 & $4 \times 6-4^{\prime} \times 9^{\prime}$ & 2,1298 & $6 \times 9-3^{\prime} \times 7^{\prime}$ & 0,7370 \\
\hline $4 \times 7-2^{\prime} \times 3^{\prime}$ & 94,6692 & $3 \times 4-2^{\prime} \times 4^{\prime}$ & 2,1285 & $4 \times 6-4 \times 7$ & 0,7366 \\
\hline $3 \times 7-7^{\prime} \times 8^{\prime}$ & 94,3242 & $5 \times 8-4^{\prime} \times 9^{\prime}$ & 2,1262 & $8 \times 9-4 ’ x 9^{\prime}$ & 0,7364 \\
\hline $3 \times 7-1 ' \times 3 '$ & 94,3030 & $4 \times 8-2^{\prime} \times 4^{\prime}$ & 2,1260 & $6 \times 9-2^{\prime} \times 7^{\prime}$ & 0,7363 \\
\hline $1 \times 2-2^{\prime} \times 7^{\prime}$ & 94,2707 & $5 \times 6-1 ' x 4^{\prime}$ & 2,1245 & $5 \times 6-1^{\prime} \times 77^{\prime}$ & 0,7360 \\
\hline $1 \times 2-2^{\prime} \times 9^{\prime}$ & 94,2709 & $3 \times 5-4^{\prime} \times 9^{\prime}$ & 2,1230 & $6 \times 7-1^{\prime} \times 7^{\prime}$ & 0,7358 \\
\hline $1 \times 7-1^{\prime} \times 2 '$ & 94,2709 & $5 \times 6-3^{\prime} \times 4^{\prime}$ & 2,1215 & $4 \times 9-2^{\prime} \times 3^{\prime}$ & 0,7357 \\
\hline $1 \times 7-2^{\prime} \times 9^{\prime}$ & 94,2707 & $4 \times 7-4^{\prime} \times 8^{\prime}$ & 2,1202 & $4 \times 6-7^{\prime} \times 99^{\prime}$ & 0,7353 \\
\hline $2 \times 5-7^{\prime} \times 9^{\prime}$ & 94,2707 & $2 \times 5-4^{\prime} \times 5^{\prime}$ & 2,1193 & $4 \times 9-4{ }^{\prime} \times 9^{\prime}$ & 0,7352 \\
\hline $3 \times 5-7^{\prime} \times 9^{\prime}$ & 94,2707 & $1 \times 5-4^{\prime} \times 9^{\prime}$ & 2,1185 & $6 \times 9-1 ' x 7^{\prime}$ & 0,7352 \\
\hline $3 \times 5-1 ' \times 9$ ' & 94,2707 & $2 \times 5-4^{\prime} \times 9^{\prime}$ & 2,1175 & $5 \times 6-3^{\prime} \times 7^{\prime}$ & 0,7349 \\
\hline $3 \times 7-1^{\prime} \times 5{ }^{\prime}$ & 94,2707 & $5 \times 6-4^{\prime} \times 6{ }^{\prime}$ & 2,1162 & $3 \times 6-4^{\prime} \times 7^{\prime}$ & 0,7347 \\
\hline $1 \times 4-2^{\prime} \times 8^{\prime}$ & 94,2377 & $5 \times 8-4^{\prime} \times 5^{\prime}$ & 2,1157 & $6 \times 9-7^{\prime} \times 99^{\prime}$ & 0,7346 \\
\hline $4 \times 7-5^{\prime} \times 8^{\prime}$ & 94,1802 & $4 \times 6-3^{\prime} \times 4^{\prime}$ & 2,1125 & $4 \times 8-4^{\prime} \times 9^{\prime}$ & 0,7346 \\
\hline $1 \times 4-3^{\prime} \times 8^{\prime}$ & 94,1497 & $5 \times 9-4^{\prime} \times 5^{\prime}$ & 2,1115 & $5 \times 7-2^{\prime} \times 8^{\prime}$ & 0,7344 \\
\hline $1 \times 4-2^{\prime} \times 5^{\prime}$ & 94,1470 & $2 \times 4-4^{\prime} \times 7^{\prime}$ & 2,1085 & $3 \times 6-7^{\prime} \times 9^{\prime}$ & 0,7343 \\
\hline $4 \times 7-2^{\prime} \times 5^{\prime}$ & 94,1467 & $4 \times 6-4^{\prime} \times 8^{\prime}$ & 2,1082 & $5 \times 6-7^{\prime} \times 9^{\prime}$ & 0,7342 \\
\hline $4 \times 7-3^{\prime} \times 5^{\prime}$ & 94,0910 & $2 \times 5-4^{\prime} \times 7^{\prime}$ & 2,1070 & $5 \times 9-2^{\prime} \times 4^{\prime}$ & 0,7342 \\
\hline
\end{tabular}


Tabela 5 - Agrupamento dos cruzamentos pelo método de TOCHER

Table 5 - Grouping of the crossings for the method of TOCHER

\begin{tabular}{|c|c|}
\hline $\begin{array}{l}\text { Grupo } \\
\text { Group }\end{array}$ & $\begin{array}{c}\text { Cruzamentos } \\
\text { Crosses }\end{array}$ \\
\hline 1 & $12^{\prime}, 13^{\prime}, 15^{\prime}, 19^{\prime}, 14^{\prime}$ e $28^{\prime}$ \\
\hline 2 & $16^{\prime}, 22^{\prime}, 33^{\prime}, 55^{\prime}, 38^{\prime}, 66^{\prime}$ e $18^{\prime}$ \\
\hline 3 & 17 ', 24', 37' e 64' \\
\hline 4 & $21^{\prime}, 31^{\prime}, 51^{\prime}$ e $47^{\prime}$ \\
\hline 5 & $23^{\prime}, 35^{\prime}, 59^{\prime}$ e $45^{\prime}$ \\
\hline 6 & $25^{\prime}, 39^{\prime}, 56^{\prime}, 99^{\prime}$ e $48^{\prime}$ \\
\hline 7 & $26^{\prime}, 42^{\prime}$ e $74^{\prime}$ \\
\hline 8 & $27^{\prime}, 44^{\prime}$ e $78^{\prime}$ \\
\hline 9 & $32^{\prime}, 53^{\prime}$ e $96^{\prime}$ \\
\hline 10 & $34^{\prime}, 57^{\prime}, 69^{\prime}$ e $91^{\prime}$ \\
\hline 11 & $36^{\prime}$ e $62^{\prime}$ \\
\hline 12 & $41^{\prime}$ e 72 ' \\
\hline 13 & $43^{\prime}, 76^{\prime}, 52^{\prime}$ e $94^{\prime}$ \\
\hline 14 & $46^{\prime}$ e $83^{\prime}$ \\
\hline 15 & $49^{\prime}, 89^{\prime}$ e $82^{\prime}$ \\
\hline 16 & 54’ e 98' \\
\hline 17 & $71^{\prime}, 81^{\prime}$ e $73^{\prime}$ \\
\hline 18 & $95^{\prime}$ e 97 ' \\
\hline 19 & $58^{\prime}$ e $86^{\prime}$ \\
\hline 20 & $63^{\prime}$ e $77^{\prime}$ \\
\hline 21 & $29^{\prime}$ e $79^{\prime}$ \\
\hline 22 & $61^{\prime}$ e $84 '$ \\
\hline 23 & $92^{\prime}$ e $93^{\prime}$ \\
\hline 24 & $65^{\prime}$ e $75^{\prime}$ \\
\hline 25 & 87 \\
\hline 26 & $88^{\prime}$ \\
\hline 27 & 85 \\
\hline 28 & $11^{\prime}$ \\
\hline 29 & $68^{\prime}$ \\
\hline
\end{tabular}

\section{Conclusões}

As médias das características são fundamentais em programa de seleção para compor cruzamentos entre linhas, para melhoria de características tanto na matrizes, como no frango de corte.

A predição de híbridos é fonte importante de informação complementar para o melhorista.

Neste estudo, existe similaridade entre os cruzamentos onde se utiliza a linha 1 (linha macho) como progenitor, o que não acontece com as demais linhas.

\section{Literatura Citada}

ARUNACHALAM, V. Genetic distance in plant breeding. The Indian Journal of Genetic and Plants Breeding, v.41, n.2, p.226-236. 1981.

COSTA, C.A. Pontos críticos do manejo de matrizes. In: Manejo de matrizes. Campinas: Fundação APINCO de Ciência e Tecnologia Avícolas, 1994. 10p.

CRUZ, C.D.; REGAZZI, A.J. Modelos biométricos aplicados ao melhoramento genético. Viçosa, MG: Universidade Federal de Viçosa, 1994. 390p.

CRUZ, C.D. Aplicação de algumas técnicas multivariadas no melhoramento de plantas. Piracicaba: Escola Superior de Agricultura "Luiz de Queiroz", 1990. 188p. Tese (Doutorado em Genética e Melhoramento) - Escola Superior de Agricultura "Luiz de Queiroz", 1990.

CRUZ, C.D. Programa GENES - Aplicativo computacional em genética e estatística. Viçosa, MG: Universidade Federal de Viçosa, 1997. 442p.

FREITAS, R.T.F. Estudo da divergência genética de suínos em cruzamentos, utilizando-se técnica de análise multivariada. Viçosa, MG: Universidade Federal de Viçosa, 1996. 152p. Tese (Doutorado em Genética e Melhoramento) Universidade Federal de Viçosa, 1996.

PIASSI, M. Avaliação do desempenho de linhagens de postura mantidas na Universidade Federal de Viçosa, em competição com marcas comerciais. Viçosa, MG: Universidade Federal de Viçosa, 1994. 86p. Dissertação (Mestrado em Genética e Melhoramento) - Universidade Federal de Viçosa, 1994.

RAO, C.R. Advanced statistical methods in biometric research. New York: John Wiley \& Sons, 1952. 389p.

TORRES, R.A. Conseqüências do melhoramento genético. Cadernos Técnicos da Escola de Veterinária da Universidade Federal de Minas Gerais, n.17, p.73-77, 1996. 